\title{
Providing Alternative Measures For Addressing Adverse Drug-drug Interactions
}

\author{
Silva, António \\ pg25307@alunos . uminho.pt \\ Satoh, Ken \\ jneves@di.uminho.pt
}

\author{
Oliveira, Tiago \\ toliveira@di.uminho.pt \\ Novais, Paulo \\ pjon@di.uminho.pt
}

\begin{abstract}
Clinical Practice Guidelines (CPGs) are documents used in daily clinical practice that provide advice on how to best diagnose and treat diseases in the form of a list of clinical recommendations. When simultaneously applying multiple CPGs to patients, this can lead to complex multiple drug regimens (polypharmacy) with the potential for harmful combinations of drugs. The need to address these adverse drug events calls forth for systems capable of not only automatically represent the common potential conflicts or interactions that can happen when merging CPGs but also systems capable of providing conflict-free alternatives. This paper presents a solution that represents CPGs as Computer-Interpretable Guidelines (CIGs) and allows the automatic identification of drug conflicts and the provision of alternative measures to resolve these conflicts.
\end{abstract}

\section{Introduction}

Drug-drug interactions occur when an effect of one drug alters the effect of another co-administrated drug [6]. Such interactions are common in multimorbid patients since they suffer multiple health conditions and need the application of different disease-specific treatment plans. To help ease the burden of health care professionals, Clinical Practice Guidelines (CPGs) were developed in order to provide patient-specific advice. These documents accumulate and reflect knowledge on how to best diagnose and treat diseases in the form of a list of clinical recommendations. When treating multimorbid patients, health care professionals need to retrieve clinical recommendations from multiple chronic disease CPGs. The result is concurrent execution of treatment recommendations from different CPGs, which may cause conflicts. From the combination of these recommendations, several problems can happen, such includes adverse drug events, and increased treatment complexity and cost of treatment [2].

Several projects were developed not only to formally represent CPGs as Computer Interpretable-Guidelines (CIGs). Through the formalisation of CPGs 
as CIGs it is possible to develop decision support systems that offer a better possibility of affecting clinical behaviour in relation to narrative documents of the corresponding text versions. The representation of CPGs in digital format can have distinct benefits over paper-based CPGs in that they increase flexibility, minimise errors, and generalise the use of CPGs across institutions. However, few works address potential conflicts or interactions that can happen when merging CIGs.

Although some approaches offer ways to represent the conflicts and interactions between concurrent CIGs, they lack in: provision of alternative measures to resolve conflicts in treatment plans, dynamic search for solutions to conflicts outside the existing knowledge base, and provision of methods to rank and select treatment plans. The first contribution of this work is a characterisation of existing approaches to handle the combination of CIGs, especially for multimorbid patients. Then, it presents a system that automatically identifies recommendation interactions, conflicts, and provides alternative measures (mainly in the form of alternative drug recommendations) that resolve the identified conflicts.

The paper is organised as follows. Section 2 describes related work regarding systems for combining CIGs. Section 3, presents an architecture for combining CIGs as well as the contributions for the deployment of CIGs in Clinical Decision Support Systems (CDSSs). Section 4 describes the functionalities supporting care with a case example of how the system processes drug-drug interactions and provides alternative measures. Finally, Section 5 presents conclusions about the work developed so far and future work considerations.

\section{Related Work}

Several formalisms are proposed in the literature that are aimed to represent the conflicts and interactions among different CPGs. They provide various methods to model the conflicts of CPGs into their knowledge base. In this section, we describe different systems that automatically identify the possible interactions between concurrent CPGs for multimorbid patients.

Wilk et al. [13] represent CIGs as an activity graph. They use constraint logic programming and combines it with constraint satisfaction problems. By using constraint logic programming, they identify and mitigate possible adverse interactions when applying multiple guidelines on the same patient, namely identifying conflicts associated with potentially contradictory and adverse activities. They provide notification features that inform the healthcare professionals about the possible conflicts during the definition of the treatment plans. This approach provides automatic identification of conflicts, however, it depends on the availability of the information in the knowledge base about the conflicts between both CIGs in the form of constraints and of pre-existing operators to mitigate these conflicts. This requires substantial manual effort for combining CIGs. Thus, in order to provide automatic identification and resolution of conflicts, solutions need to be defined in a medical background knowledge as protocol-dependent rules/constraints. 
Lopez et al. [8] used a rule-based methodology in order to identify and reconcile drug conflicts between recommendations of two concurrently executed CIGs. In order to provide a treatment plan without interactions, they utilise a standard terminology called ATC (Anatomical Therapeutic Chemical Classification System for drugs). The outputted treatment plan comprises a set of ATC-codes of medicines, without interactions, which should be prescribed. They manually build knowledge units for the pairwise combination of diseases in their knowledge base. These knowledge units rely on the existence of drug-drug interactions, the presence of a drug which is adverse to a specific disease (drugdisease interaction) and the absence of a necessary medicine for a combination of diseases. Although this approach can only combine CPGs pairwise, it is possible to achieve a final treatment plan for any number of CIGs by combining a pair of CIGs into a general CIG and then combining the latter with a new CIG. This approach requires significant manual effort as each combination has to be hardcoded.

OntoMorph [5] represent guidelines as a collection of ontologies. They use information such as the general domain, the mappings between CPGs and decision rules for simultaneous execution of CPGs that are provided by domain experts. Based on these ontologies, they developed a system capable of merging two concurrent CIGs into a co-morbid personalised guideline. By representing the CIGs as ontologies, it allows retrieving the clinical tasks from the CPG and converts them to computer-interpretable rules in Ontology Web Language (OWL). Using ontologies is one of the possible solutions to CPG representation. It allows the representation of declarative knowledge (medical statements and propositions) and procedural knowledge (workflow structures and actions) as rules. Ontomorph also has a merging representation ontology, which allows capturing merging criteria to achieve the combination of CIGs. By using Semantic Web Rule Language(SWRL) rules, they can identify potential conflicts during the merging process. Since all conditions need to be defined in their model during the merging process, this increases the effort to maintain the system up-to-date and reduces the possibility of sharing knowledge. In their work, some of the identified limitations were not yet entirely addressed, such as potential contradictions between rules, the scalability of the merging model to combine several CIGs, and how the ontology/rules are maintained up-to-date.

The Transition-based Medical Recommendations for Interactions (TMR4I) model is a model that automatically infers the interaction between recommendations [14] by using meta-rules for the identification and reconciliation of three categories of drug conflicts using SPARQL queries (SPARQL is a W3C-standard for semantic queries). Using meta-rules allows defining how a conflict is identified and how similar drugs without interactions and conflicts can be selected as alternatives. The categories of conflicts within CPGs are repetition interactions, contradiction interactions and alternative interactions. The model was extend in [14] with additional interaction types and several measures such as deontic strength, causation belief, and belief strength. This work provides only a representation of conflicts but does not afford reasoning or any form of decision support. 
The limitations of above-mentioned approaches include: restrictions in the number of CIGs that can be combined, necessity of all solutions to be available in a knowledge base, and decidability of reasoning mechanisms. In the approaches that require hard-coded solutions, if a conflict that is not accounted for in the knowledge base appears, the reasoning component will not be able to provide a response. Also worth mentioning is that current approaches do not provide support for ranking sets of guideline recommendations that are consistent.

\section{An Architecture for CIG Management with CIG Interaction Detection and Resolution}

The present work not only aims to provide recommendations to support medical decision-making but also to represent automatically the conflicts and interactions that can happen when merging CIGs. In this work we focus on drug-drug interactions and propose a system capable of automatically identifying recommendation (drug) interactions using existing terminology services, namely the RxNorm API [7]. Once interactions are identified, we provide alternative measures, i.e., alternative drugs to the ones recommended that would not cause any conflict, through a mitigation function. This function calculates the solutions for the identified conflicts using different mitigation principles such as similarity between drugs or user preferences. The architecture is shown in Figure 1. This architecture is a three-level solution that encompasses the following stages for the CIG deployment: representation of CPGs in CIGs, identification of recommendation interactions and provision of recommendation alternatives in case that some recommendations, when applied together, are adverse. The following sections explains the architecture that integrates these three levels.

\subsection{Representation of CPGs in CIGs}

The work described herein uses the CompGuide ontology to represent CPGs in the form of a task network. The CompGuide ontology [10] contains different types of clinical tasks such as Question, Action, Decision, End, Plan and provides different types of clinical constraints expressed in the form of conditions on the patient's state, such as TriggerConditions, PreConditions and Outcomes. Through the utilisation of object properties to connect instances of the sub-classes of the clinical tasks, it is possible to define the relative order between tasks. In the Compguide ontology, it is possible to define sequential tasks, parallel tasks, and alternative tasks. Moreover, it provides a model of temporal representation [11] that aims to represent the temporal constraints placed on clinical tasks. This model represents temporal constructors on the execution of tasks such as Durations, Repetitions, Periodicities, Waiting Times and Repetition Conditions and temporal constraints about the state of a patient. To acquire and represent CPGs we use the CompGuide plugin which provides information step-by-step on how to fill the data for the guideline entries [4]. This plugin performs the role of managing the creation and editing of CIGs. 


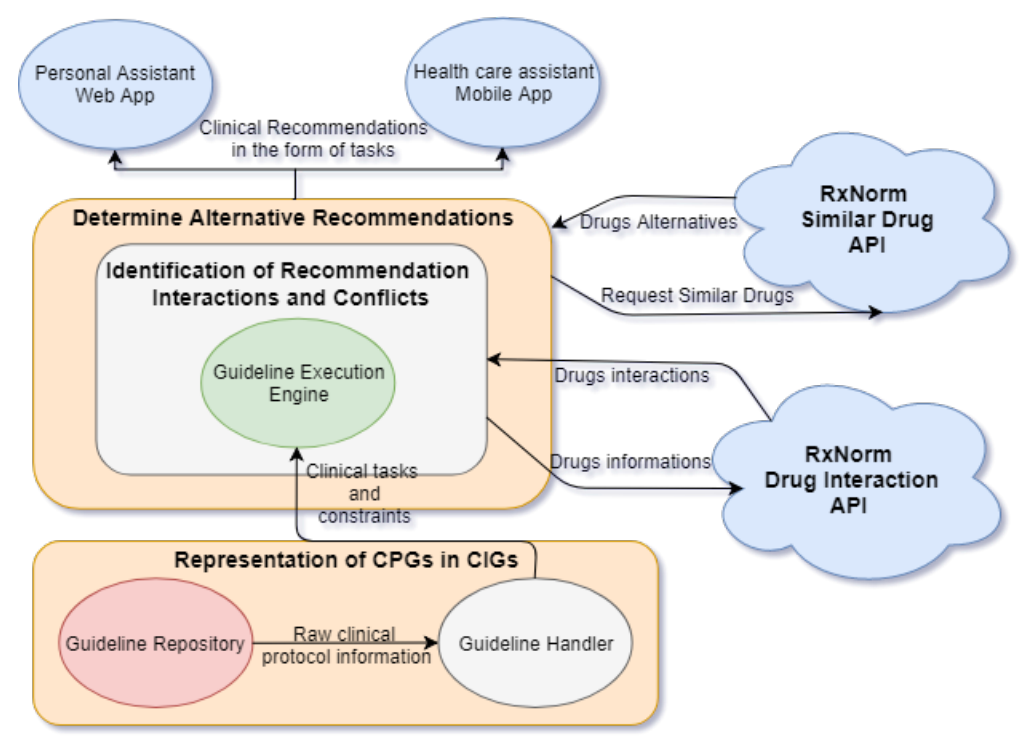

Figure 1: Architecture of CompGuide system

The output of guideline encoding is a will CIG that will be saved in the Guideline Repository. This component is responsible for keeping different CIGs defined according to the CompGuide ontology. The Guideline Handler is responsible for managing the access to CIG recommendations in the Guideline Repository, providing the clinical tasks and constraints placed on the tasks to the Guideline Execution Engine.

\subsection{Identification of Recommendation Interactions}

The Guideline Handler provides clinical task details to the Guideline Execution Engine in order to produce recommendations. This component provides information about temporal constraints on the execution of the clinical tasks. Using this information, the Guideline Execution Engine produces task enactment times, and by using RxNorm Interaction API, it determines if drug-drug interactions exist. As before-mentioned, the clinical tasks are defined, in the CompGuide ontology, by a set of subclasses. One of these subclasses is Action, in which it is possible to define a collection of drugs that require to be prescribed. The Action class has several subtypes of actions, namely exams, procedures, medication recommendations and simple recommendations [11]. The Recommendation medication provides a set of drugs that can be prescribed and is used by Guideline Execution Engine to determine drug-drug interactions. By using the RxNorm interaction API, it is possible to determine drug-drug interactions, without the need to manually define drug interactions in the knowledge base. Its interaction API [7] uses two data sources, ONCHigh and DrugBank 
and provide information such as source name, severity and description of the interaction. Thus, the Guideline Execution Engine processes all the clinical tasks that are being executed, retrieves all drugs and for each pair of drugs calls the RxNorm Interaction API to obtain the severity and description of the interaction.

\subsection{Generating Alternative Recommendations}

After processing all drug-drug interactions between concurrently executed clinical recommendations, the alternative measures are evaluated by the system. If an adverse drug event exists, the systems automatically try to find alternative recommendations to resolve the conflict. Through a mitigation function, the system determines which alternative recommendations are advised. This function encompasses a set of steps that include the following:

- Step 1: The system tries to find if it is possible to get alternative recommendations, i.e., alternative drugs, in the guidelines. For each specific guideline recommending a drug, the system calculates if an alternative recommendation exists within the guideline. If it is not possible to retrieve the alternative recommendation, the system moves to step 2;

- Step 2: Using the RxNorm API, the system tries to find conflict-free alternative drugs. The system provides these alternative drugs by determining the set of alternatives that have the high similarity score concerning the given drugs. The RxNorm API provides the similarity score. If there are no alternative drugs the system moves to step 3;

- Step 3: The system evaluates all possible solutions using Multiple-criteria Decision Analysis (MCDA). Since drug-drug interactions are yielding multiple solutions with conflicting objectives, it is useful to score the solutions. The patient and physician score all possible solutions, so this is a shared patient-clinician evaluation supported by the system.

In step 1, the system tries to resolve the conflicts by analysing within the guideline the different task alternatives. In this particular case, if alternative tasks (recommending alternative drugs) exist in the guideline, the system tries to retrieve them. Then, it gets all the recommended drugs of the alternative tasks and tries to find if drug-drug interactions exist in them, by calling RxNorm Interaction API for each pairwise drugs of the task. In step 2, a ranking of alternative drugs is produced based on the similarity score provided by the RxNorm API. The similarity score between drugs is a score that determines the similarity between drugs. Thus, the system calls the RxNorm API to get alternative drugs for the given conflicted drugs and calculates the highest similarity score for the alternative drugs and for each alternative with the higher score it tries to encounter conflict-free drug. If there is a conflict, the system finds the next alternative with the higher score, if there is no conflict, it stores the alternative in the knowledge base. Table 1 presents the MCDA approach for 
step 3. This approach uses a value measurement model where for each criterion the patient assigns a score. The objective of this model is constructing and comparing numerical scores (overall value) to identify the degree to which one decision alternative is preferred over another. Each alternative to be scored is a combination of drugs. The system automatically defines the criteria by which decision-makers should orient. Thus, when an adverse drug event occurs, and the system moves to step 3, the criteria established are: severity of disease for which drugs are advised, adverse drug-drug interactions and expected outcomes for the drug application. The criteria are defined on the basis of some types of health care decisions that are implemented in projects such as EMAs BenefitRisk Methodology Project [12] and shared patient-clinician decision [3]. The total score for each alternative is obtained by multiplying a numerical score for each option on a given criterion by the relative weight for the criterion and later summing these weighted scores. Thus, the total score is provided by the following expression:

$$
f(n)=\sum_{n=1}^{n} S^{n} * W \text { eight } C^{n},
$$

where $n$ is the number of solutions to be scored, $S^{n}$ a score of a specific solution and $W$ eight $C^{n}$ relative weight for a specific criterion.

The relative weight for the criterion is a value defined by the healthcare professional. This value is requested before starting the evaluation of the solutions. After getting the user scores, the system determines the total score of each solution by using the aforementioned equation. Thus, the total scores of each solution are made available through the Personal Assistant Web App and Healthcare assistant Mobile App, presenting the selected solution. Moreover, after processing the constraints of clinical tasks, determining the interactions between drugs and their alternatives, the clinical recommendations are made available in before-mentioned assistants. In this assistants, it is possible to visualise the clinical recommendations that currently are being applied to the patient, in a calendar and timeline view. Thus, each clinical recommendation can have a set of drugs or alternative solutions that were previously evaluated and scored by decision makers.

Table 1: Assessment of all possible solutions. The symbol $C$ indicates a certain criterion to be evaluated for a given solution $\alpha$. $S$ means the score of the solution.

\begin{tabular}{|c|c|c|c|c|}
\hline \multirow[b]{2}{*}{ Solutions $(\alpha)$} & \multicolumn{3}{|c|}{ Criterion $(C)$} & \multirow[b]{2}{*}{ Total Score } \\
\hline & $\mathrm{C}^{1}$ & $\ldots$ & $\mathrm{C}^{n}$ & \\
\hline$\alpha^{1}$ & $\mathrm{~S}^{1} C^{1}$ & $\ldots$ & $\mathrm{S}^{1} C^{n}$ & $\mathrm{f}(1)=\sum_{n=1}^{1} S^{1} *$ Weight $C^{n},(2)$ \\
\hline$\alpha^{n}$ & $\mathrm{~S}^{n} C^{n}$ & $\ldots$ & $\mathrm{S}^{n} \mathrm{C}^{n}$ & $\mathrm{f}(\mathrm{n})=\sum_{n=1}^{n} S^{\dddot{n}} * W$ eight $C^{n}$ \\
\hline
\end{tabular}




\section{Case Example}

This section describes how CompGuide processes the interactions between drugs given a case test example. For this purpose, we used two CIGs based on the NCCN Clinical Practice Guideline for Prostate Cancer [9] and the IDF Clinical Practice Recommendations for managing Type 2 Diabetes [1]. These guidelines were a comprehensive case study since it was possible to test several aspects of the deployment of CIGs. However, in this section, we only address the conflicts between recommendations from many guidelines and provision of alternative recommendations using step 2 described in section 3.2.

For demonstration purposes, we will consider two recommendations from the mentioned guidelines. The first one, named recommendation A belongs to the guideline for managing Type 2 Diabetes: "Apply insulin 0.2 units $/ \mathrm{kg}$ and titrate once weekly at one unit each time during six months to achieve a target fasting blood glucose between 3.9 and $7.2 \mathrm{mmol} / \mathrm{L}(70$ and $130 \mathrm{mg} / \mathrm{dL}) "$. The second recommendation, named recommendation B belongs to the guideline for prostate cancer: "Apply goserelin, leuprolide, histrelin $180 \mathrm{mg} / \mathrm{m} 2$ or Triptorelin $100 \mathrm{mg} / \mathrm{m} 2$ as part of Androgen Deprivation Therapy".

Recommendation A has the action apply insulin, a periodicity value of 1 with a temporal unit of week, a duration value of six, the respective temporal unit of month and medication recommendation insulin. In this case, starting on the $18^{\text {th }}$ of July of 2018 the system will create one event for each week with a duration of one day, during 6 months. The expected conclusion of this task will be on the $18^{\text {th }}$ of January of 2019. As for recommendation B, the action to apply goserelin, leuprolide, histrelin or triptorelin can be identified, with a duration value of 1 and temporal unit of day, starting and finishing on the $18^{\text {th }}$ of July of 2018. The recommendation medications are goserelin, leuprolide, histrelin and Triptorelin. The application tries to provide alternative drugs to address the identified conflicts, by calling RxNorm API and will provide alternative medicines according to step 2, as described in section 3.2. Also, in this step the system calculates a ranking of conflict-free alternative drugs, using the similarity score provided by RxNorm API. The ranking of alternative drugs is calculated by comparing similarity scores and sorting in descending order the medicines according to this similarity values. For the alternative drug (drug provided by RxNorm) with a higher score, the system determines if it is conflict-free over the prescribed drugs. If there is a conflict, the system finds the next alternative with the higher similarity score, if there is no conflict, it stores the alternative in the database and displays the alternative drug as the selected solution.

In the work described herein, we provide a system that automatically identifies conflicts and interactions between drugs for many guidelines. Comparing with the works of Jafarpour et al. (2013) [5], Wilk et al. (2017) [13] and LpezValverd et al. (2013) [8], where conflicts are defined as constraints in the knowledge base having to be manually specified, CompGuide uses existing terminology services that aggregate different drug sources such as ONCHigh and DrugBank. Thus, through the reuse and integration of existing terminology services such as RxNorm, it is possible to identify conflicts and interactions automatically, 
without the need to manually define them in the knowledge base. Therefore, using existing terminology services and resorting to external knowledge sources is one of the possible solutions for the limitation mentioned above.Another solution concerns the use of meta-rules such as those used by the TM4I model. Meta-rules can be reused since they can be applied to many CIGs, and conflicts do not need to be manually identified for each guideline, because they can be automatically derived from the guideline representation. However, the bottleneck will be in converting guidelines to computer-interpretable rules. Besides, these systems do not consider aspects such as decision-making. In most cases, there are several alternatives that can lead to conflicting objectives by the decision makers. In other cases, it is necessary to decide which recommendation we want to choose, or which recommendation, in the case at hand, is less adverse. For this specific case, we provide an MCDA approach that allows to evaluate all possible solutions based on conflicting criteria.

\section{Conclusions and Future Work}

The application of multiple clinical protocols individually can result in complex multiple drug regimens (polypharmacy) with the potential for harmful combinations of drugs. Some of the studied approaches are unable to detect the conflicts for combinations of protocols automatically. Other approaches cannot propose alternative measures that would resolve the conflicts. Other CIG models require all the possible conflicts and their solutions to be available in a knowledge base. Moreover, they cannot lead with cases where decision makers have conflicting solutions or cannot decide on the best treatment alternatives.

As a means to solve these issues, we provide a multiple criteria decisionmaking approach for not only assessing the benefit-risk of applying the recommendations but also getting patient preferences on best treatment alternatives. This allows to evaluate all possible solutions and to specify different criteria to solve conflicts with medical recommendations, beyond the simple comparison of drug interactions. We also offer a system that allows to combine the knowledge of several guidelines and to identify drug interactions and conflicts among many recommendations automatically. Comparing with some of the studied systems, the CompGuide has an additional advantage since it presents a method to automatically identify drug-drug conflicts among many recommendations, without a necessity of manually define them in the knowledge base. Also, when decision makers have conflicting solutions and cannot decide on the best treatment alternatives, the CompGuide presents an approach that allows to evaluate all possible solutions and to specify different criteria to solve conflicts with medical

recommendations. As future work, we intend to make a proper assessment of the fitness of the system for CIG deployment, by performing a study involving physicians interacting with the system in the clinical environment. In this ways, it is possible to analyse if the system meets the requirements of health professionals and if it is user-friendly. 


\section{Acknowledgements}

This work has been supported by FCT Fundao para a Cincia e Tecnologia within the Project Scope: UID/CEC/00319/2019.

\section{References}

[1] Aschner, P.: New idf clinical practice recommendations for managing type 2 diabetes in primary care (2017)

[2] Boyd, C.M., Darer, J., Boult, C., Fried, L.P., Boult, L., Wu, A.W.: Clinical practice guidelines and quality of care for older patients with multiple comorbid diseases: implications for pay for performance. Jama 294(6), 716$724(2005)$

[3] Dolan, J.G., Boohaker, E., Allison, J., Imperiale, T.F.: Patients preferences and priorities regarding colorectal cancer screening. Medical Decision Making 33(1), 59-70 (2013)

[4] Gonçalves, F., Oliveira, T., Neves, J., Novais, P.: Compguide: Acquisition and editing of computer-interpretable guidelines. In: World Conference on Information Systems and Technologies. pp. 257-266. Springer (2017)

[5] Jafarpour, B., Abidi, S.S.R.: Merging disease-specific clinical guidelines to handle comorbidities in a clinical decision support setting. In: Conference on Artificial Intelligence in Medicine in Europe. pp. 28-32. Springer (2013)

[6] Kennedy, C., Brewer, L., Williams, D.: Drug interactions. Medicine (United Kingdom) 44(7), 422-426 (2016), http://dx.doi.org/10.1016/j. mpmed.2016.04.015

[7] Liu, S., Ma, W., Moore, R., Ganesan, V., Nelson, S.: Rxnorm: prescription for electronic drug information exchange. IT professional 7(5), 17-23 (2005)

[8] López-Vallverdú, J.A., Riaño, D., Collado, A.: Rule-based combination of comorbid treatments for chronic diseases applied to hypertension, diabetes mellitus and heart failure. In: Process Support and Knowledge Representation in Health Care, pp. 30-41. Springer (2013)

[9] Mohler, J.L., Lee, R.T., Antonarakis, E.S., Armstrong, A.J., D'Amico, A.V., Davis, B.J., Dorf, T., Eastham, J.A., Ellis, R., Enke, C.A., Farrington, T.A.: National Comprehensive Cancer Network - Prostate Cancer. Tech. rep., National Comprehensive Cancer Network (2018), http: //linkinghub.elsevier.com/retrieve/pii/B9780323358682000803

[10] Oliveira, T., Novais, P., Neves, J.: Representation of clinical practice guideline components in owl. In: Trends in Practical Applications of Agents and Multiagent Systems, pp. 77-85. Springer (2013) 
[11] Oliveira, T., Silva, A., Neves, J., Novais, P.: Decision support provided by a temporally oriented health care assistant. Journal of medical systems 41(1), 13 (2017)

[12] Phillips, L.D., Fasolo, B., Zafiropoulos, N., Beyer, A.: Is quantitative benefit-risk modelling of drugs desirable or possible? Drug discovery today: technologies 8(1), e3-e10 (2011)

[13] Wilk, S., Michalowski, M., Michalowski, W., Rosu, D., Carrier, M., Kezadri-Hamiaz, M.: Comprehensive mitigation framework for concurrent application of multiple clinical practice guidelines. Journal of biomedical informatics 66, 52-71 (2017)

[14] Zamborlini, V., Da Silveira, M., Pruski, C., ten Teije, A., Geleijn, E., van der Leeden, M., Stuiver, M., van Harmelen, F.: Analyzing interactions on combining multiple clinical guidelines. Artificial intelligence in medicine 81, 78-93 (2017) 